\title{
Uma Abordagem para Recuperação e Recomendação Automática, Dinâmica e Não Determinística de Objetos de Aprendizagem Baseada em Estilos de Aprendizagem, em Metadados no Padrão IEEE LOM e em Elementos de Web Semântica
}

\author{
Vitor C. de Carvalho ${ }^{1}$ \\ Orientador: Fabiano A. Dorça ${ }^{1}$ \\ ${ }^{1}$ Faculdade de Computação - Universidade Federal de Uberlândia (UFU) \\ Uberlândia - MG - Brasil \\ vitorcarvalho@comp.ufu.br, fabianodor@ufu.br
}

\begin{abstract}
Studies show that students tend to have a better learning when the content presented to then is personalized. Thus, automatic content adaptation in education systems is an area that has been the subject of several studies. However, you must have a sufficient amount of content to be customized so that it is efficient. This paper presents an analysis of an approach of recommendation and Learning Objects recovery, based on the IEEE LOM standard in Felder Learning Styles and the OAI-PMH Protocol.
\end{abstract}

Resumo. Estudos mostram que estudantes tendem a ter melhores resultados no aprendizado quando o conteúdo lhe é apresentado de forma personalizada. Dessa forma, a adaptação automática de conteúdo em sistemas de ensino é uma área que vem sendo alvo de diversos estudos. Porém, é necessário ter uma quantidade suficiente de conteúdo a ser personalizado para que esta seja eficiente. Este trabalho apresenta uma análise de uma abordagem de recomendação e recuperação de Objetos de Aprendizagem, baseado no padrão IEEE LOM, nos Estilos de Aprendizagem de Felder e no Protocolo OAI-PMH.

\section{Introdução}

Para auxiliar no ensino e aprendizado dos estudantes, surgiram diversos trabalhos que criam técnicas para serem usadas em Sistemas de Tutores Inteligentes (STI), que fazem uso de Objetos de Aprendizagem (OA) para auxiliar no processo de ensino. OAs esses que são materiais, digitais ou não, que tem como objetivo serem usados no aprendizado de algum conteúdo, como por exemplo, vídeos, textos, áudios, livros e apostilas.

OAs devem ter também como característica, a capacidade de serem usados, reusados e referenciados por outros sistemas além dos que foram originados [Wiley 2000]. Para que esses atributos estejam presentes, é necessário que eles possuam um padrão.

Este trabalho apresenta uma abordagem de Recomendação de OAs automática, dinâmica e não-determinística, que usa como base o padrão IEEE 2004 LOM (Learning Object Metadata) 1484.12.1 [Hodgings and Duval 2002] para conseguir uma boa eficiência, se tratando de acerto de objetos recomendados ao perfil certo. 


\section{CBIE-LACLO 2015}

Anais dos Workshops do IV Congresso Brasileiro de Informática na Educação (CBIE 2015)

Porém, um padrão para os OAs não é suficiente, pois existe a necessidade de se mapear o perfil do estudante no sistema. Para tal serviço, utiliza-se neste trabalho o modelo de Estilos de Aprendizagem (EA) criado por Felder and Silverman 1988.

O modelo de EA de Felder é amplamente utilizado nesta linha de pesquisa, por representar com muita eficácia os estudantes reais em um modelo computacional facilmente entendível pelos STIs.

Graf et al. 2010 cita que atualmente, criar abordagens eficientes que forneçam adaptatividade, considerando os EA do estudante e tendo em mente das incertezas e dificuldades existentes na modelagem do estudante e processo de ensino é um dos grandes desafios da atualidade. Assim, o modelo de EA utilizado neste trabalho se difere do modelo convencional pois será representado numa versão não-determinística e sim probabilística, onde os estilos dividirão o todo da dimensão a que pertencem no modelo de Felder.

Criar conteúdo para os mais diversos perfis de estudantes existentes é uma tarefa cara a ser realizada, portanto, o trabalho também traz um modelo para recuperação de OAs de diversos Repositórios de Objetos de Aprendizagem (ROA) públicos.

Para manter a compatibilidade entre os dois sistemas, os metadados recuperados no modelo de recuperação também devem estar no padrão LOM, portanto este é o foco do trabalho. Além disso, foram estudados conceitos de Web Semântica para se aproveitar de suas vantagens. O modelo também conta com o uso do protocolo Open Archive Initiave Protocol for Harvesting Metadata (OAI-PMH) ao recolher os metadados dos ROAs.

O restante do artigo encontra-se organizado da seguinte maneira: na Seção 2, são discutidos trabalhos relacionados; na Seção 3, são apresentados os principais conceitos utilizados no trabalho; na Seção 4, é descrita a abordagem proposta; na Seção 5, são apresentados os resultados dos experimentos realizados; e, por fim, na Seção 6, são feitas as considerações finais e apresentados os trabalhos futuros.

\section{Trabalhos Relacionados}

Em busca de vencer o desafio de se obter uma recomendação de conteúdo eficiente, diversas pesquisas são iniciadas todo o tempo. Um exemplo é o trabalho de Cazella et al. 2012 que utiliza o conceito de competências para realizar a recomendação de OAs. Um dos pontos negativos é a grande quantidade de intervenções humanas que devem ser realizadas durante o processo.

Ribeiro et al. 2014 apresentaram uma pesquisa, que tinha como objetivo, o aprimoramento do Ambiente Virtual de Aprendizagem (AVA) do Moodle [Rice 2011]. A abordagem que seguiram foi a de integrar o AVA com a plataforma Google na Obtenção de conteúdo. Este trabalho, entra também no âmbito de pesquisa para recuperação de conteúdo e não somente recomendação.

A Recuperação de Conteúdo da Web é outro desafio a se enfrentar, pois, o desgaste proporcionado na fabricação dos Objetos de Aprendizagem é grande. Dessa forma, possuir um módulo em seu STI que faça a recuperação de forma eficiente, pode otimizar o tempo gasto pelo instrutor, além de auxiliar o estudante que desejar encontrar material de estudos. 
Vian et al. 2010 por exemplo, criaram um modelo para indexação e recuperação eficiente de $\mathrm{OA}$ em repositórios baseando-se no paradigma de agentes inteligentes e no emprego de sistemas ontológicos para descrição do domínio do conhecimento nos mecanismos de busca.

Estes trabalhos, de forma geral, utilizam repositórios de objetos de aprendizagem como fonte de dados. Mas existem trabalhos como o de Ribeiro et al. 2014 que tentam utilizar o conteúdo recuperado de motores de busca em geral e não apenas o conteúdo recuperado no formato de OA.

Este trabalho, apresenta uma abordagem hibrida entre os métodos de recomendação e os de recuperação de objetos de aprendizagem. A abordagem de recomendação de OA leva em consideração os Estilos de Aprendizagem do Estudante de forma probabilística, pois acredita-se que este modelo traz vantagens sobre o modelo convencional de utilização dos EA, como citado por Silva and Dorça 2014.

Além disso, a abordagem conta com a utilização de padrões e modelos bem estruturados. O IEEE LOM, por exemplo, é utilizado em sua forma original, que possui um grande poder de representação para os OAs e os torna capazes de ter em seus metadados, a capacidade de serem replicados e reutilizados independente do sistema em que estão integrados.

O outro exemplo é o modelo de EA de Felder and Silverman 1988, que tem todas suas dimensões aproveitadas no trabalho. A utilização do modelo completo, garante ao trabalho um grande poder de representação dos perfis dos estudantes, por se tratar de um modelo muito bem definido e amplamente reconhecido.

Para a abordagem de recuperação, o trabalho traz um modelo conciso e trabalhado em cima também de conhecimentos comuns à área. Para garantir uma recuperação eficiente e genérica, ou seja, que cumpra o trabalho a qual foi designado, podendo este recuperar material independente da área, foram aproveitados os conceitos definidos pela Web Semântica.

E para a realização da recuperação propriamente dita, foi escolhida a utilização do protocolo OAI-PMH, protocolo este que é bem definido pela organização OAI. A utilização deste protocolo, nos garantiu uma ampla diversidade de ROA públicos que possuem serviços específicos para o mesmo, o que soma mais um ponto positivo para a abordagem proposta.

\section{Referencial Teórico}

Serão apresentados nesta seção, algumas considerações sobre conhecimentos necessários para a compreensão deste trabalho.

\subsection{Modelo de Felder-Silverman}

Felder and Silverman 1988 perceberam que cada pessoa tem sua forma de receber e processar as informações que recebe, a isso, eles chamaram de Estilos de Aprendizagem (EA). Estes EA, não são excludentes entre si, dessa forma o que acontece de fato é que cada pessoa possui uma afinidade maior a um estilo do que a outro.

Para melhor entendimento do significado de cada dimensão, utilizando-se da Tabela 1, Franzoni et al. 2008 explica sobre cada um dos EA. 
CBIE-LACLO 2015

Anais dos Workshops do IV Congresso Brasileiro de Informática na Educação (CBIE 2015)

Tabela 1. Tabela de estilos do Modelo Felder.

\begin{tabular}{|c|c|c|}
\hline $\begin{array}{l}\text { Dimensao do Estilo de } \\
\text { Apredizagem }\end{array}$ & Tipo & Descrição \\
\hline \multirow{2}{*}{ Percepção } & Sensitivo (S) & $\begin{array}{l}\text { Sabe lidar com fatos, dados puros e experimentos, é paciente com } \\
\text { os detalhes, mas não gosta de complicaçốes. }\end{array}$ \\
\hline & Intuitivo (I) & $\begin{array}{l}\text { Sabe lidar com principios e teorias, fica facilmente entediado } \\
\text { quando são apresentados muitos detalhes e tende a aceitar } \\
\text { complicações. }\end{array}$ \\
\hline \multirow{2}{*}{ Canal de Entrada } & Visual (Vi) & $\begin{array}{l}\text { Se lembra bem do que viu: imagens, diagramas, tabelas temporais, } \\
\text { filmes, etc. }\end{array}$ \\
\hline & Verbal (Ve) & Se lembra do que escutou, leu ou disse. \\
\hline \multirow{2}{*}{ Processamento } & Ativo (A) & Aprende bem em grupo e com o material que tem em mãos. \\
\hline & Reflexivo (Re) & $\begin{array}{l}\text { Aprende melhor quando pensa e reflete sobre a informação dada a } \\
\text { ele. Trabalha melhor sozinho ou com no máximo mais uma pessoa. }\end{array}$ \\
\hline \multirow{2}{*}{ Entendimento } & Sequencial (Seq) & $\begin{array}{l}\text { Segue um raciocínio linear quando está resolvendo problemas e } \\
\text { pode trabalhar com material específico uma vez que compreende } \\
\text { ele parcialmente ou superficialmente. }\end{array}$ \\
\hline & Global (G) & $\begin{array}{l}\text { Tem grandes intuiçôes sobre a informação, pode ter dificuldade de } \\
\text { expor como chegou a certo resultado, precisa de uma visão geral. }\end{array}$ \\
\hline
\end{tabular}

Este modelo é muito usado hoje por dar a base para representar o perfil dos estudantes em Sistemas Adaptativos e Inteligentes para Educação (SAIE).

\section{2. eXtensible Markup Language}

eXtensible Markup Language (XML) [Bray et al. 1998] é uma linguagem de marcação recomendada pela $\mathrm{W} 3 \mathrm{C}$ para criação de documentos organizados hierarquicamente, como em uma árvore de dados. Ela é chamada "extensível" pois permite que sejam definidos elementos utilizados na marcação, ao contrário do HTML, por exemplo.

Devemos ressaltar a importância do XML em trabalhos como este, pois, seu alto poder de representação nos permite definir conceitos de forma que o computador possa ler sem intervenção humana, algo que não seria possível com uma linguagem humana convencional, como o inglês ou o português.

Toda a representação dos padrões utilizados neste trabalho, bem como o resultado de todas as comunicações de dados realizadas, foi feita com a utilização de documentos XML.

\subsection{Web Semântica}

A Web Semântica é a parte da Web que provê métodos e padrões que nos permitem atribuir significado as informações presentes na Web convencional. Suas funcionalidades e tecnologias estão dispostas por suas camadas, apresentadas na Figura 1.

As funcionalidades que a Web Semântica nos provê são interessantes por auxiliar tanto na escolha dos dados recuperados, quanto na utilização da informação recuperada na Web [Koivunen and Miller 2001, Yu 2011]. Dentre as suas tecnologias mais importantes, estão o Resource Description Framework (RDF) da camada RDF que nos e a Web Ontology Language (OWL) [Shadbolt et al. 2006] da camada de Ontologias [Primo et al. 2010]. 
CBIE-LACLO 2015

Anais dos Workshops do IV Congresso Brasileiro de Informática na Educação (CBIE 2015)

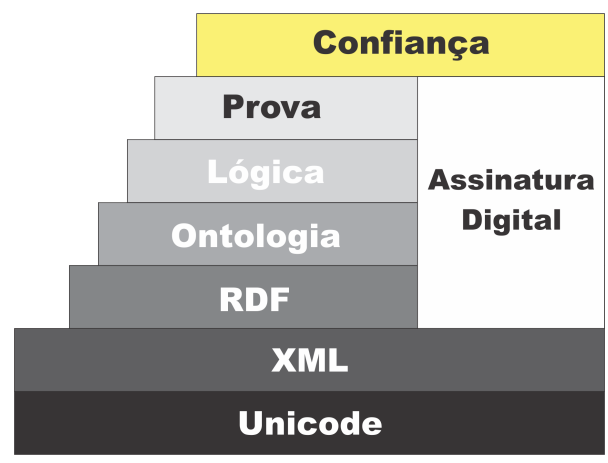

Figura 1. Camadas da Web Semântica. (Adaptado de [Yu 2011])

\subsection{Repositório de Objetos de Aprendizagem}

Os autores de OAs os disponibilizam na Web para que potenciais interessados possam reutilizá-los. Normalmente, os serviços que os disponibilizam são chamados de Repositórios de Objetos de Aprendizagem (ROAs) [Mohan 2005].

Esses ROAs possuem interfaces de usuário e arquiteturas que os tornam fáceis de usar, e permitem vários níveis de interatividade. [Lehman 2007]. Eles são comumente um banco de dados eletrônico que acomoda uma coleção de OAs, permitindo sua melhor organização e melhorando a eficiência em suas reutilização e colaboração.

\subsection{Open Archives Initiative - Protocol for Metadata Harvesting}

O protocolo OAI-PMH foi criado pelo OAI, e tem como função auxiliar os desenvolvedores a realizar Harvesting de metadados em repositórios remotos, ou seja, trazer os metadados de objetos virtuais de Data Providers para um diretório local.

Neste trabalho, os principais verbos utilizados do protocolo foram: ListMetadataFormats, para verificar quais os formatos de metadados cada repositório escolhido disponibiliza para seus OAs, o ListRecords, para listar os OAs recuperados no repositório e o GetRecord que nos traz os metadados de um único OA, no formato desejado.

Para uma especificação mais detalhada dos parâmetros e da lista de erros disponíveis para cada verbo do protocolo, visite OAI 2015.

\subsection{Padrão LOM}

Para que os OAs possam ser recuperados, eles devem possuir algumas características: interoperabilidade, acessibilidade e durabilidade. Interoperabilidade é a capacidade de se compartilhar um mesmo OA entre sistemas com ferramentas distintas. Acessibilidade é a capacidade de utilizar os OAs de forma remota. Durabilidade é a possibilidade de se utilizar um OA sem reprojeto ou recriação, mesmo quando houver mudança na base tecnológica. Além disso, existe ainda uma característica que afeta diretamente a criação, o uso e reuso de um OA: a chamada granularidade [Duval and Hodgins 2003].

Para que essas características se mantenham, é necessário que haja um padrão, como o LOM [Hodgings and Duval 2002]. O LOM é composto por 68 elementos hierarquizados, divididos em 9 categorias, que descrevem a semântica dos metadados de um OA. 
CBIE-LACLO 2015

Anais dos Workshops do IV Congresso Brasileiro de Informática na Educação (CBIE 2015)

\section{Abordagem Proposta}

Podemos separar a metodologia utilizada no desenvolvimento do trabalho nas seguintes fases:

- Estudo do padrão IEEE LOM e do Modelo de EA de Felder.

- Estudo da Web Semântica e de suas camadas.

- Criação de um modelo de Recomendação eficiente.

- Estudo sobre Repositórios de Objetos de Aprendizagem.

- Criação de um modelo de recuperação de metadados de Repositórios Remotos.

- Análise do modelo geral apresentado.

- Criação do sistema e realização de testes.

Definir as fases de estudo para o trabalho foi importante para dar continuidade a ele. Seguindo a metodologia proposta, iniciou-se o desenvolvimento das pesquisas. Este que é descrito ao decorrer da seção.

Utilizando a semântica das informações providas pela Web Semântica, é possível melhorar a eficácia de alguns pontos de aplicações. A partir das informações que conseguimos através dos aquivos XML que recebemos, podemos realizar todo o processamento necessário.

Arquivos XML os quais, entende-se, sempre que citado o termo "metadados", refere-se a sua representação por meio deles, e não por outro tipo de persistência, como Banco de Dados, por exemplo.

O primeiro módulo da aplicação, referente recomendação de objetos de aprendizagem. A partir e campos específicos do LOM, foi feito um mapeamento [Resende and Dorça 2013] e foram definidas regras para abstração do entendimento. Estas regras estão descrita no trabalho de de Carvalho and Dorça 2014.

A cada regra interpretada como verdadeira, é acrescentado um ponto ao EA em questão, $X$. O cabeçalho da regra especifica o EA a ser incrementado, enquanto o corpo da regra, especifica os campos a serem analisados.

Por exemplo, um OA que seja descrito como \{"Formato: áudio", "Interatividade: Expositivo", "Tipo de Recurso: Diagrama", "Dificuldade: complexo" $\}$, obtendo uma pontuação de 3 pontos no mapeamento para o EA "Sensitivo" e de 1 ponto no EA "Intuitivo", ou seja, esse OA é 75\% "Sensitivo" e 25\% "Intuitivo".

Então, após adquiridas as dimensões a que pertence o estudante, e as do mapeamento do OA, é realizado um cálculo de distância vetorial, feito pela técnica de City Block, e essa distância se refere a pontuação que o OA recebe em relação ao EA do estudante. No ranqueamento, os OAs com menor pontuação (ou seja, menor distância) são os que aparecem no topo da lista.

Importante afirmar aqui, como pode ser observado, que neste trabalho, não existe um módulo de análise do Modelo de Estudante, portanto, para a utilização eficiente da recomendação, é necessário que se detecte estes modelos de outra forma conhecida, como a de Silva and Dorça 2014.

O segundo módulo da aplicação, tem como objetivo recuperar os metadados de repositórios remotos e trazê-los para um diretório local. Nele, a partir de um domínio conhecido, pode-se realizar a busca por Objetos de Aprendizagem que contenham informações 
de um assunto desejado e a partir dessa busca, escolher quais acrescentar ao repositório local..

Para recuperarmos OAs, é preciso criar uma forma de conexão com o Repositório de Objetos. O OAI-PMH foi utilizado neste trabalho para realizar a conexão com o repositório e recuperar seus metadados. Entretanto, por ser muito simples e com funções básicas, não possui um filtro de resultados obtidos via palavras-chave, trazendo assim, a complexidade desta funcionalidade para a implementação do software que responsável pela recuperação dos metadados.

Escolhido um ROA, deve-se conseguir sua URL de conexão no servidor OAIPMH. Com a URL, o serviço ListRecords do OAI-PMH foi utilizado para recuperar uma lista dos metadados disponíveis no repositório e o verbo GetRecord para capturar um metadado escolhido. $\mathrm{O}$ foco da escolha de repositórios de objetos de aprendizagem neste trabalho, foram os repositórios de acesso público [Group et al. ].

\section{Experimentos}

Para realização dos testes de Recomendação, foram empregados OA's fictícios. Todas as 16 combinações possível de EA's foram testadas [Dorça et al. 2013]. Cada qual com diferentes variações em seus percentuais para o mesmo conjunto de OA, de modo a se analisar melhor o resultado obtido.

Os resultados, em geral, foram limitados pelo pouco número de OA's disponíveis para os testes. No entanto, os resultados mostraram-se promissores. Notou-se que os piores resultados foram obtidos no ranqueamento de OA's com poucas informações, situação em que os EA's adquirem valores muito altos na sua classificação. Apesar disso, os valores dos OA's situados nas primeiras posições do ranque comportaram-se conforme esperado.

Apesar de se manter a predominância dos EA's, variações na mesma fazem com que alguns OA's variem consideravelmente no ranque. Como o modelo não leva em consideração a predominância dos OA's, mas sim a distância entre seus valores e os dos EA do estudante, um novo calculo é feito separadamente para cada recomendação realizada, já que as "notas" dadas a cada OA difere entre um estudante e outro. Esse tipo de comportamento pode ser observado na Figura 2, em variações nos perfil do estudante (a), (b) ou (c) - alteram o ranqueamento dos OA's e, consequentemente, a recomendação.

Quando encontrados repositórios que possuem formato IEEE LOM, porém, em sua maioria estão totalmente incompletos. O repositório Kobe Shoin Women's University Repository, por exemplo, possui em seus servidores, metadados no padrão IEEE LOM, mas os utiliza apenas como agregador de palavras-chave, como apresentado na Figura 3.

Outro problema comum, é encontrar repositórios que possuem compatibilidade o formato IEEE LOM para seus metadados, mas não possuem nenhum objeto salvo no padrão. O repositório The Learning Edge é um exemplo disso. Ao realizar o teste de validação, existe a compatibilidade com o IEEE LOM, mas não há nenhum objeto disponível neste formato.

\section{Conclusão}

Com base na realização de experimentos, a proposta de recomendação de OA foi validada e mostrou-se potencialmente eficaz, podendo este ser utilizada em ambientes virtuais de 
CBIE-LACLO 2015

Anais dos Workshops do IV Congresso Brasileiro de Informática na Educação (CBIE 2015)
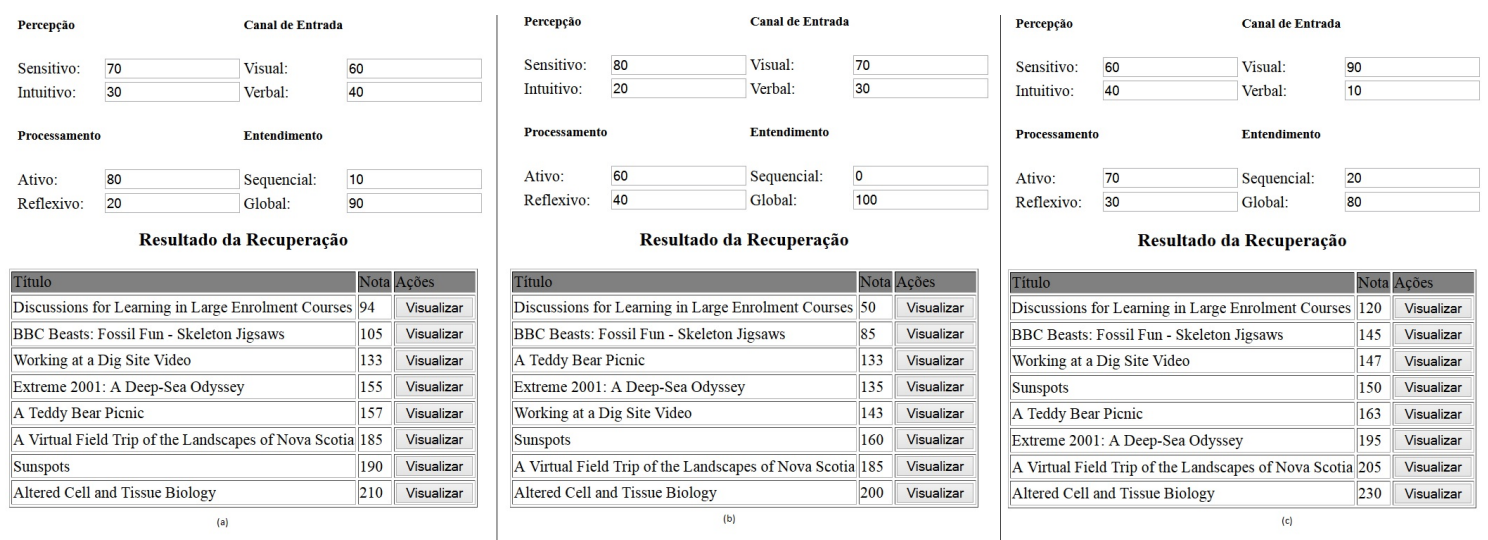

Figura 2. Exemplo de variação no ranque conforme valores configurados para o perfil do estudante.

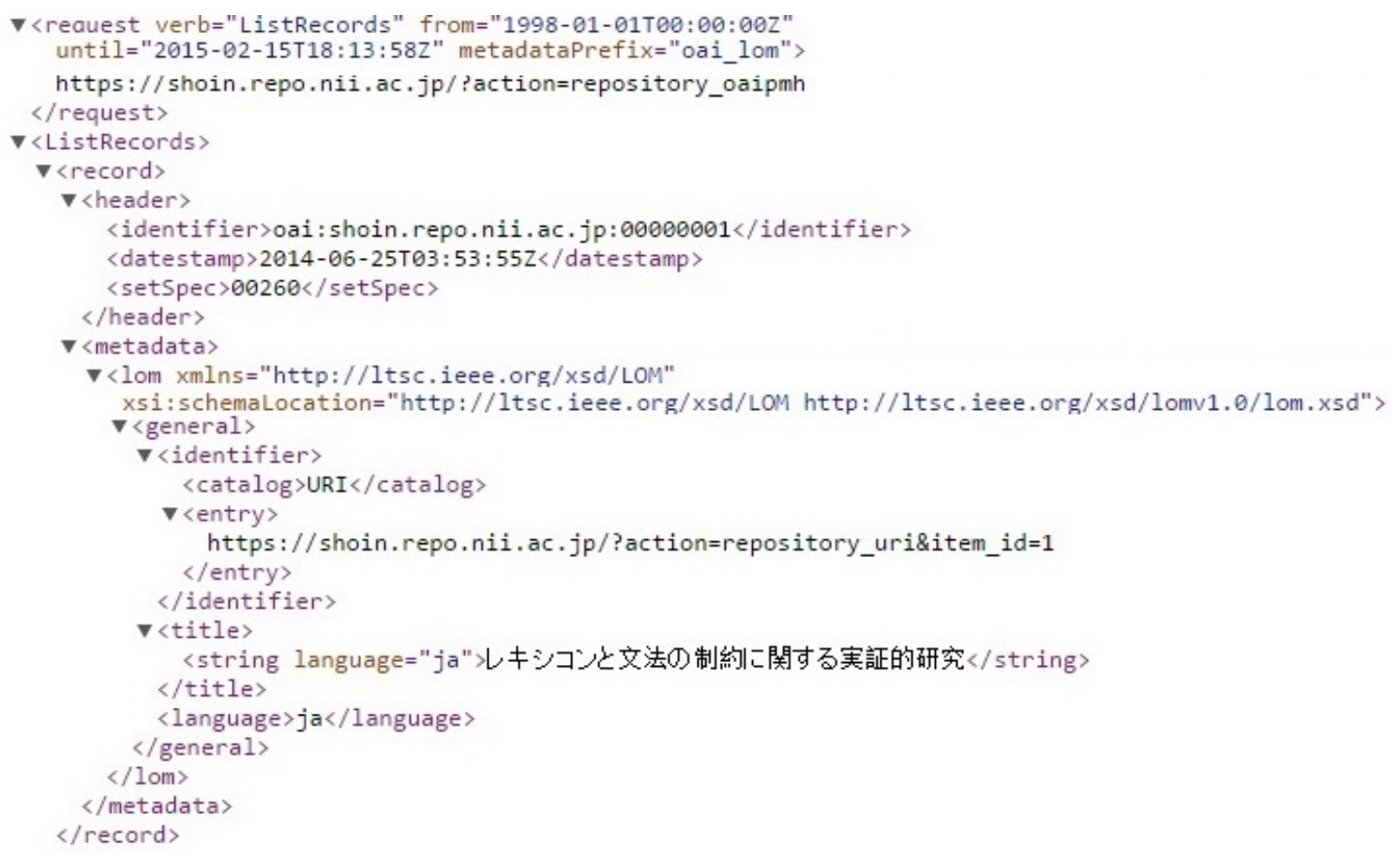

Figura 3. Lista de Metadados Dispostos pelo Repositório "Kobe Shoin Women's University Repository". 


\section{CBIE-LACLO 2015}

Anais dos Workshops do IV Congresso Brasileiro de Informática na Educação (CBIE 2015)

aprendizagem que forneçam um modelo do estudante baseado em EA's [Felder and Silverman 1988]. Para isso, porém, depende de certo compromisso por parte dos autores de conteúdo no preenchimento dos metadados que descrevem os OA's, dado que alguns campos são obrigatórios para se obter precisão no ranqueamento.

O módulo de recuperação demonstrado pelos resultados apresentados, possui uma forma de trabalho válida e funcional. Porém, a utilização do módulo de recuperação fica restrita no aspecto da dificuldade de se encontrar repositórios de objetos de aprendizagem que possuem o padrão de metadados no formato desejado.

Como trabalhos futuros, pretende-se integrar o modelo a um ambiente virtual de aprendizagem para testes com OA's e estudantes reais. Para tanto, espera-se criar uma abordagem que discuta uma forma de se aproveitar dos metadados no modelo Dublin Core utilizado em grande parte dos repositórios públicos convertidos nos campos desejados do IEEE LOM.

E ainda, criar ferramentas de desenvolvimento se utilizem melhor das capacidades e do poder de representação que a Web Semântica oferece, fazendo uso de Ontologias como um conceito facilitador na recuperação do conteúdo.

\section{Agradecimentos}

$\mathrm{O}$ autor agradece as agências brasileiras de pesquisa CNPq, CAPES, FAPEMIG e a PROPP/UFU pelo apoio concedido a este trabalho.

\section{Referências}

Bray, T., Paoli, J., Sperberg-McQueen, C. M., Maler, E., and Yergeau, F. (1998). Extensible markup language (xml). World Wide Web Consortium Recommendation REC-xml19980210. http://www. w3. org/TR/1998/REC-xml-19980210.

Cazella, S. C., Behar, P., Schneider, D., da Silva, K. K., and Freitas, R. (2012). Desenvolvendo um sistema de recomendação de objetos de aprendizagem baseado em competências para a educação: relato de experiências. In Anais do Simpósio Brasileiro de Informática na Educação, volume 23.

de Carvalho, V. C. and Dorça, F. A. (2014). Uma abordagem para recomendação automática e dinâmica de objetos de aprendizagem baseada em estilos de aprendizagem e em metadados no padrão ieee lom. DesafIE - III Workshop de Desafios da Computação Aplicada à Educação nos Anais do Congresso da Sociedade Brasileira de Computação, pages $636-645$.

Dorça, F. A., Lima, L. V., Fernandes, M. A., and Lopes, C. R. (2013). A new approach to discover students learning styles in adaptive educational systems. Revista Brasileira de Informática na Educação, 21(01):76.

Duval, E. and Hodgins, W. (2003). A lom research agenda. In WWW (Alternate Paper Tracks).

Felder, R. M. and Silverman, L. K. (1988). Learning and teaching styles in engineering education. Engineering education, 78(7):674-681.

Franzoni, A. L., Assar, S., Defude, B., and Rojas, J. (2008). Student learning styles adaptation method based on teaching strategies and electronic media. In Advanced 


\section{CBIE-LACLO 2015}

Anais dos Workshops do IV Congresso Brasileiro de Informática na Educação (CBIE 2015)

Learning Technologies, 2008. ICALT'08. Eighth IEEE International Conference on, pages 778-782. IEEE.

Graf, S., Ives, C., et al. (2010). A flexible mechanism for providing adaptivity based on learning styles in learning management systems. In Advanced Learning Technologies (ICALT), 2010 IEEE 10th International Conference on, pages 30-34. IEEE.

Group, S. W. et al. Jisc: Dublin core scholarly works application profile http://www. ukoln. ac. uk/repositories/digirep/index.

Hodgings, W. and Duval, E. (2002). Ieee ltsc learning object meta-data lom_1484_12_1_v1_final_draft.

Koivunen, M.-R. and Miller, E. (2001). W3c semantic web activity. Semantic Web KickOff in Finland, pages 27-44.

Lehman, R. (2007). Learning object repositories. New directions for adult and continuing education, 2007(113):57-66.

Mohan, P. (2005). Learning object repositories. In Informing Science and IT Education Joint Conference.

OAI (2015). OAI for beginners tutorial, 2015. http://www.oaforum.org/tutorial/. Acesso em 25/02/2015, url = http://www.oaforum.org/tutorial/.

Primo, T. T., Vicari, R. M., and da Silva, J. M. C. (2010). Rumo ao uso de metadados educacionais em sistemas de recomendação. In Anais do Simpósio Brasileiro de Informática na Educação.

Resende, D. T. G. R. and Dorça, F. A. (2013). Personalização em sistemas de ensino à distância com base no mapeamento de estilos de aprendizagem em objetos de aprendizagem.

Ribeiro, F. A. A., Fonseca, L. C. C., and de Sousa Freitas, M. (2014). Sistema de recomendação de documentos na internet utilizando meta-busca para o moodle. DesafIE - III Workshop de Desafios da Computação Aplicada à Educação nos Anais do Congresso da Sociedade Brasileira de Computação, pages 618 - 626.

Rice, W. (2011). Moodle 2.0 E-Learning Course Development. Packt Publishing Ltd.

Shadbolt, N., Hall, W., and Berners-Lee, T. (2006). The semantic web revisited. Intelligent Systems, IEEE, 21(3):96-101.

Silva, D. H. and Dorça, F. A. (2014). Uma abordagem automática para personalização do processo de ensino baseada em estilos de aprendizagem em sistemas adaptativos e inteligentes para educação a distância. Revista Brasileira de Informática na Educação, 22(02):1.

Vian, J. et al. (2010). Sistema multiagente para indexação e recuperação aplicado a objetos de aprendizagem.

Wiley, D. (2000). Learning object design and sequencing theory.

Yu, L. (2011). A developer's guide to the semantic web. Springer. 